\title{
La réécriture du Narcisse de Pierre Jean Jouve et les miroirs du Moi dans Le Monde désert
}

\section{Fabrizio Impellizzeri}

\section{(2) OpenEdition}

Journals

\section{Édition électronique}

URL : http://journals.openedition.org/rief/234

DOI : $10.4000 /$ rief.234

ISSN : 2240-7456

\section{Éditeur}

Seminario di filologia francese

\section{Référence électronique}

Fabrizio Impellizzeri, « La réécriture du Narcisse de Pierre Jean Jouve et les miroirs du Moi dans Le Monde désert ", Revue italienne d'études françaises [En ligne], 3 | 2013, mis en ligne le 15 décembre 2013, consulté le 01 mai 2019. URL : http://journals.openedition.org/rief/234; DOI : 10.4000/rief.234

Ce document a été généré automatiquement le 1 mai 2019.

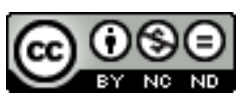

Les contenus de la RIEF sont mis à disposition selon les termes de la Licence Creative Commons Attribution - Pas d'Utilisation Commerciale - Pas de Modification 4.0 International. 


\title{
La réécriture du Narcisse de Pierre Jean Jouve et les miroirs du Moi dans Le Monde désert
}

\author{
Fabrizio Impellizzeri
}

$1 \quad$ Les figures des dieux et des héros de la mythologie grecque jouent un rôle manifeste dans la littérature en incarnant ce lieu où le désir peut se laisser présenter sans voiles, sans masques ni tabous ${ }^{1}$. À cet égard, le personnage mythique de Narcisse $^{2}$ est celui qui évoque le plus fidèlement cette tradition du simulacre et traduit dans l'art et l'écriture toute forme de révélation par une projection de soi sur la surface lisse du tableau ${ }^{3}$ ou de la page. C'est pourquoi, au moment de l'essor des sciences psychologiques en France ${ }^{4}$, il suscite de nouvelles interprétations, et il acquiert une incontestable importance dans le panorama littéraire français dès la fin du XIX ${ }^{e}$ siècle. D'une manière plus ou moins évidente, Narcisse se transforme ainsi en un véritable objet de culte pour nombre de romanciers qui inaugurent le nouveau siècle imprégné des premières études freudiennes. Cette génération d'écrivains, qui suivent le symbolisme, trouvent dans cette figure énigmatique et « contemplative » l'emblème même du conflit de l'identité et de la dualité spécifique de la nature humaine. Pierre Jean Jouve ${ }^{5}$ n'échappe pas à cette tradition, et récupère par le biais du personnage de Jacques dans Le Monde désert ${ }^{6}$ (1927) ce mythe du Narcisse qui lui fournit une nouvelle approche pour interpréter la modernité où l'homme se regarde « au miroir » de ses fragilités.

\section{Le Narcisse jouvien au reflet de ses « sources »}

2 Pour Jouve, la première apparition de la relecture contemporaine du mythe est celle que nous retrouvons dans Le Traité du Narcisse (1891) qu'un jeune André Gide, son ami ${ }^{7}$, nous présente sous la forme d'un sujet inquiet, incertain, fils du spleen, inexorablement érodé par l'ennui. Ce Narcisse, tout comme celui de notre auteur, est follement désireux de pouvoir se contempler et part, comme la légende l'indique, en quête d'un miroir8. L'écrit 
autofictionnel de Gide se propose ainsi principalement comme un lieu où l'artiste imaginaire est non seulement l'écrivain, mais un écrivain qui cherche par l'écriture de l'aveu à donner une image psychologique de lui. A priori, la réécriture du Narcisse se révèle tout d'abord comme une figure de la duplication de soi dans une écriture vaine; mais elle assume, chez les auteurs qui suivent, une consistance plus moderne, plus complexe et articulée. Cette génération, qui s'apprête à lire les ouvrages de Freud, dresse une image " positive » du Narcisse engendrée par le conflit des instances psychiques. En 1921, Pierre Jean Jouve épouse en seconde noce la psychanalyste Blanche Reverchon, célèbre traductrice de Sigmund Freud et amie de Jacques Lacan. Dès lors, il devient un des premiers écrivains à se tourner vers la psychanalyse et à montrer l'importance de l'inconscient dans la création artistique. Il aborde les textes de Freud avec l'aide de sa femme, jusqu'à en subir une influence déterminante dans son écriture, comme nous allons le montrer.

3 Le terme "narcissisme » a été emprunté par Freud à un texte de Paul Näcke datant de 1889, Die sexuellen Perversitäten, dans lequel ce dernier propose ce mot pour désigner un comportement dans lequel un individu traite son propre corps comme le corps d'un objet sexuel. Le mot apparait ainsi pour la première fois chez Freud en 1910, pour rendre compte du choix d'objet chez les homosexuels9. Comme nous le montrerons, Jouve ne suit pas Freud dans son interprétation du narcissisme comme «libido du moi $»^{10}$ d'un sujet incapable de se tourner vers un objet extérieur ${ }^{11}$, mais il en exploite surtout l'« état narcissique premier [...] [un] état primitif, qu'il nomme alors de narcissisme primaire, [...] caractérisé par l'absence totale de relation à l'entourage, par une indifférenciation du moi et du ça, et trouverait son prototype dans la vie intra-utérine [...] $»^{12}$. De la sorte, Jouve s'oriente plutôt vers la conception qui est exposée dans l'ouvrage Pour introduire le narcissisme (1914) où le "narcissisme [...] ne serait [plus] une perversion, mais le complètement libidinal à l'égoïsme de cette pulsion d'autoconservation dont on attribue, à juste titre, une part à chaque créature vivante $»^{13}$.

Pierre Jean Jouve invente son propre mythe de Narcisse, en s'appuyant ainsi sur des textes freudiens plus tardifs et essentiellement sur la pulsion de mort qui est centrale à l'œuvre Au-delà du principe de plaisir (1920). Comme il est évident dans Le Monde désert, « La pulsion de mort [...] représente la tendance fondamentale de tout être vivant à retourner à l'état anorganique $»^{14}$, par le suicide dans notre cas. Jacques ne pourra échapper à ce désir de se retrouver dans un état primitif, dans la substance aquatique spécifique de la réalité intra-utérine, dans ce rassurant « sentiment océanique $»^{15}$ qui le libèrera de tous ses troubles.

5 Le personnage de Jouve, Jacques, recherche, davantage qu'un amour de soi, un oubli mystique de soi, une fusion dans le " grand-tout ». Jouve peut ainsi lier son intérêt pour la psychanalyse et sa mystique religieuse. Il affirme que «[...] l'homme moderne a découvert l'inconscient et sa structure; il y a vu l'impulsion de l'éros et l'impulsion de la mort, nouées ensemble, et la face du monde de la Faute, je veux dire du monde de l'homme, en est définitivement changée $\aleph^{16}$. Jacques de $\operatorname{Todi}^{17}$, personnage principal du roman, représente bien aux yeux de Pierre Jean Jouve, la figure « gidienne » du Narcisse. Être de l'altérité et du double, Jacques est essentiellement un homme qui est gouverné sans cesse par deux instincts contradictoires: le sens de la vie ou de la sexualité, c'est-à-dire l'épanouissement de ses désirs, et le sens de la mort qui traduit le sentiment de la faute qui le hante perpétuellement ${ }^{18}$. Le héros du Monde désert n'est autre qu'une pure projection d'un $\mathrm{Moi}^{19}$ que la conscience condamne à un irréparable état de déchirement. 
[...] le Moi jouvien. Différent de la personne P. J. Jouve, ce moi, est une entité spirituelle, conçue comme support ou centre d'une pensée qui, au plan de l'expression ou de l'œuvre ne cesse de se diviser en une multitude de facettes et de genres. [...] Dans l'optique jouvienne, l'aventure à la fois capitale et primordiale de la scission s'identifie avec l'expérience de la faute et de la chute. [...] Péché originel, luciférisme romantique et culpabilité de Dieu d'une part, ambivalence dans l'attitude en face du père et formation du sur-moi de l'autre: les théories se superposent et se côtoient sans qu'il soit possible de connaître celle qui se trouve à l'origine de ce jeu de correspondances. ${ }^{20}$

6 À l'appui de ce qui vient d'être argumenté, René Micha, dans une étude datant de 1942, nous présente un Pierre Jean Jouve qui puise dans l'inconscient la source de son écriture et estime s'y refléter. L'érotisme, l'expérience mystique, le sens de culpabilité et l' « appétit cannibale » du complexe de Narcisse qui en dérive, sont au centre de l'œuvre jouvienne et Micha nous le révèle en ces termes :

Jouve fait état des découvertes de Freud [...]. Il puise au merveilleux inconscient, assuré d'y trouver une première explication de l'homme. Mais il va plus loin. [...] Il éprouve en même temps l'expérience érotique et l'expérience mystique. [...] L'amour n'est [pour Jouve] [...] [qu'] une arme étonnante entre les mains de l'homme. L'amour plus souvent perd l'homme qu'il ne le sauve [...]. Il réveille la faute installée dans l'homme qui n'attend qu'un signe. Comme une sangsue, il est collé à l'inconscient [...]. Quelques-uns ont sondé cet abîme, commencé de dénombrer ces monstres qu'ils appellent [...] le complexe de Narcisse [...], [un véritable] appétit cannibale [...]. On [y] trouve le poète agenouillé devant la source de l'amour, il s'y mire en tremblant, inquiet et adorable et déjà il rêve de la capter. ${ }^{21}$

\section{Jacques et son Monde désert}

7 Jacques se partage toujours d'une manière obsessive et angoissante entre ses pulsions érotiques homosexuelles ${ }^{22}$ et le châtiment créé par un puissant Surmoi qui dérive principalement de son éducation protestante représentée, la plupart du temps, par la figure du père et de la religion ${ }^{23}$. C'est ainsi qu'il s'identifie et qu'il culpabilise :

\footnotetext{
Je suis un misérable, je suis un immonde, je ne mérite pas d'exister, je veux mourir, je suis un misérable, un infâme, je salis tout ce que je touche, il ne faut plus que j'existe, je suis un diffamateur, je veux mourir, je suis un monstre, un séducteur et un violateur, j'ai fabriqué des mensonges [...]. (MD, p. 60)
}

Jacques ne s'accepte donc pas et sa douleur tragique et existentielle naît principalement d'un conflit insoluble qui postule une rupture de l'unité, de son Moi, qui subit sans cesse "La force de deux êtres dont l'un dompte, domine et rétablit l'autre» ( $M D$, p. 61). Une puissante tension existe de ce fait chez notre héros entre deux forces inconciliables également autoritaires ${ }^{24}$ : le bien et le mal, l'homme et la femme, l'esprit et la chair, la lumière et les ténèbres, et enfin, d'une manière symbolique, le désert et l'eau. À cet égard, Wanda Rupolo affirme que chez Jouve « le rapprochement ou le repoussement des deux principes, entre lesquels est renfermée toute l'histoire de la vie humaine, et l'exigence de reconstituer l'unité, engendrent une intensité profondément dramatique $»^{25}$.

Dans un autre ordre, la psychanalyse encourage Pierre Jean Jouve à s'orienter vers une lecture symbolique et donc métaphorique du monde. Dans Le Monde désert, l'introspection s'installe d'emblée dans son élément naturel: la désolation. L'égarement s'efforce de répondre à la question fondamentale de l'être et devient le leitmotiv du récit. Métaphore du vide, le titre du roman évoque déjà la poignante expérience de la solitude que vit le héros homosexuel et représente, par conséquent, sa fermeture du moi au monde. Le 
désert dont nous parle Jouve est bien plus que ce lieu abandonné, solitaire, désolé, privé de végétation et infécond qui envahit notre imaginaire, il est ce « désert réel ou mythique [...] qui est toujours l'image d'une solitude morale $»^{26}$. Il symbolise également, pour le héros, la rupture des liens familiaux. Pur miroir de ce vide, le monde dans lequel évolue Jacques apparaît alors bien plus qu'« un dur pays désertique à traverser» (MD, p. 107), c'est un lieu où il est contraint à une « fausse vie » (MD, p. 106) :

Je la voyais notre vie : un dur pays désertique à traverser, rien que des arbres sans verdure, des piquants noirs, des cailloux, un soleil du diable; je marchais bravement sur ce désert [...] la marche ne me menait nulle part. [...] Le désert qui s'étendait au fond de moi. Désert sans eau, sans force, étouffant de chaleur, jamais une larme. Pas d'adoucissements [...] rien ne changerait jamais pour moi ». (MD, p. 107-108 et 110)

Le désert est aux yeux de Pierre Jean Jouve une sorte de fond de gouffre du désespoir, une étendue aride qui creuse et épuise l'âme de Jacques. Le fil conducteur du drame est le creusement laborieux et incessant que vit le héros. Une quête infinie de l'Autre qui s'exprime, tout au long du roman, par une profonde et permanente autoanalyse qui satisfait sans doute elle aussi son narcissisme mais qui accroît, en revanche, son perpétuel sentiment de la faute. Le Monde désert est ainsi un univers hostile où s'égare à jamais le héros qui s'isole et se protège volontairement de l'incompréhension et de l'intolérance du monde bourgeois et austère de Genève ${ }^{27}$. Séparé de tout et de tous, et sans doute aussi de lui-même, Jacques mélancolique ${ }^{28}$ dit : «Je suis dans le désert. Le monde se sépare de moi. À cause de mon péché. Cependant ce péché c'est mon amour, et mon amour est beau » ( $M D$, p. 49).

Ce péché, c'est son amour. L'homosexualité de Jacques n'est pas, dans l'économie de ce récit, un trait de psychologie, mais une marque symbolique de sa solitude. Incapable de partager la sexualité commune, voué à une forme d'identité que réprouve le corps social, il sera exclu de tout partenariat, de toute union viable en ce monde. [...] La singularité de Jacques présentera toujours ce mélange de bonne et de mauvaise conscience, cette alternative d'acceptation et de refus de soi-même, qui figurent la fatalité de sa solitude. ${ }^{29}$

11 Paradoxalement pour le personnage, le désert ressemble à un mirage fantasmé qui vise à la recherche d'une oasis lointaine, d'un Eden introuvable, qui puisse le rassasier de sa soif de liberté et de bonheur. Malheureusement, au-delà de toute rêverie ${ }^{30}$, l'expérience de la solitude se révèle sans cesse aux yeux de Jacques comme tragique, un miroir de l'introjection punitive ${ }^{31}$, un dédale sans fin qui le condamne déjà à l'évasion vers l'ailleurs, vers les rivages du Rhône, vers l'eau. En ce sens, par opposition à la matière aride et brûlante du désert, Jacques trouve une idée de compensation dans l'eau, réelle invitation au voyage, à quitter la terre inhospitalière pour pouvoir finalement s'y retrouver, s'y perdre, s'y noyer " océaniquement », comme nous l'avons déjà vu.

Rappelons à cet égard que le récit s'ouvre symboliquement sur le tableau de la solitude d'un enfant, Jacques de Todi âgé de neuf ans, fils d'un pasteur protestant influent de Genève $^{32}$, qui passe la plupart de son temps dans un état chronique de rêverie, d'où il ne sort que pour fuir de son quotidien familial et s'isoler longuement dans un monde tout à lui : la berge caillouteuse du lac Léman :

Lorsque le fils du pasteur Isaac de Todi sortait de son état chronique de rêverie pour constater que le monde extérieur, la « campagne » de son père à Genève, était coloré de telle ou telle façon par la pluie ou par le beau temps, selon la saison et le jour et surtout l'imprévu de son âme à lui, il aimait descendre les trois marches basses du perron, hors du triste vestibule solennel [...] On éprouvait en ce lieu un 
silence très grand et très pur, mais si l'on y réfléchissait ce silence était formé de bruits, des innombrables rumeurs de vagues qui s'abattaient doucement [...] Pour Jacques de Todi âgé de neuf ans et plein de fantaisies et de réserves mentales, le pigeonnier sans pigeons au bord du lac avait les proportions, l'aspect, la figure immense et maudite d'un Château Enchanté devant la mer [...]. (MD, p. 13-14)

Pur exercice de l'écriture narcissique, Le Monde désert est un "roman de passage » où Jouve alterne habilement les focalisations, passant pour ainsi dire de la troisième personne à la première, et restituant, de ce fait, la « parole » à son héros. Or, comme nous l'avions dit à propos de Gide, l'écriture se transforme en aveu et se manifeste comme dans un véritable journal intime ${ }^{33}$ : on voit "couler» son encre sur des pages où se réfléchissent la plupart du temps les ombres prisonnières qui caractérisent les états d'âme plutôt sombres des trois personnages: Jacques, Luc ${ }^{34}$ et Baladine (femme de Jacques).

\section{Les retrouvailles de l'Unité}

Jacques recherche, tout au long du récit, une image « autre » à aimer qui la plupart du temps s'érotise ${ }^{35}$, s'incarne ou se divinise à travers l'eau :

0 toi que j'adore et qui es venue à moi dans l'eau, qui t'es ainsi révélée pendant tant de soirs, ou qui restes voilée sous les profondeurs [...]. Le visage contemplait Jacques à travers l'eau du lac, couvert de larmes et peut-être couvert de sourires, Jacques étant le seul au monde à savoir qu'il existait, Jacques étant le visage, l'âme, l'éternité du visage et de l'amour passionné qu'un si beau visage éveille. (MD, p. 18)

L'amour narcissique et l'amour de l'autre se confondent car, tant dans la relation avec l'Autre que dans la relation avec soi-même, Jouve-écrivain ${ }^{36}$ nous donne l'impression d'être en présence à la fois de deux personnes et d'une seule. Jacques n'accomplit une incontestable révélation de sa psychose que quand il avoue sa véritable double identité : "C'est un autre que je connais bien, étranger à moi, et moi tout de même, tu comprends?» (MD, p. 81). L'identité du sujet jouvien est, en ce sens, toujours mêlée d'altérité, et l'identité, souvent sous un double aspect ${ }^{37}$, rassemble le «Je » et l'Autre sous la forme d'une confusion qui se liquéfie et matérialise à la réverbération de l'image dans l'eau. Jacques fusionne avec cet élément liquide, il entre en communion avec la matière, se fond en elle, et en devient une partie. Il «contemplait l'eau, longuement, passionnément, il y plongeait ses regards et son cœur, il devenait l'eau » (MD, p. 15).

La personnalité et le destin de Jacques se manifestent ainsi au bord du lac Léman et nous indiquent, dès les premières pages, à quel point l'eau s'installe dans sa vie comme une forme de l'abîme, un lieu de rêverie qui engendre toutes sortes de fantasmes et fantaisies. La surface miroitante du lac favorise d'étranges visions et semble absorber, en la noyant, l'imagination de l'enfant. Au fur et à mesure qu'il l'observe, l'eau prend possession de lui, l'enveloppe, et l'emmène vers une sorte de cérémonie magique de l'identification. Le regard du héros est comme hypnotisé, il suit ses mouvements, ses agitations, ses frémissements, et s'éloigne de ce fait de toute réalité «terrienne ». C'est alors que surgit dans l'eau, à moins que ce ne soit « dans l'âme de Jacques » (MD, p. 15), une surprenante apparition, une femme aux traits changeants, parfois même sans figure :

Il contemplait l'eau, longuement, passionnément, il y plongeait ses regards et son cœur [...], alors apparaissait quelquefois dans l'eau, c'est-à-dire dans l'âme de Jacques, une figure extraordinaire, très belle et rêveuse, aussi veloutée que l'eau, dont aucun trait ne demeurait fixe, mais changeait et rechangeait: un jour elle 
avait les cheveux noirs, les yeux infiniment brillants chargés de cils noirs, le visage mince et la peau brune ; un autre jour blonde et vraiment douce et rose comme une femme; l'instant d'après elle prenait un visage tragique avec des yeux et des cheveux incertains; ou même elle ressemblait à sa mère [...]. Par les soirs les plus précieux, les plus beaux, l'apparition était toute entière sans figure, sans trait que l'on pût discerner ou même imaginer, elle était sans être, et Jacques de Todi tendait son âme éperdue vers l'eau vivante afin de la recevoir. Et doucement, sans employer de mots, lui parler. (MD, p. 15-16)

17 Ainsi, l'eau et le visage sont une seule et même chose, un reflet intérieur de Jacques qui fait parler son âme. Le miroir et l'image spéculaire se confondent et le miroitement évoque l'essence double de Jacques qui se partage entre chair et esprit. L'âme trouve enfin sa voix dans cette image fuyante et trouble que le reflet lui renvoie. Elle recompose dès lors cette unité du moi et du Ça qui semblait perdue à jamais. Grâce à l'eau, comme nous l'avons suggéré plus haut, Jacques semble retrouver un équilibre personnel et une paix amniotique qui l'apaise et l'accorde définitivement à lui-même. Une image intérieure qui, dans son inconsistance, révèle toutefois un aspect obscur et caché de Jacques, et se substitue ainsi au reflet de l'apparence qu'évoquait le mythe à son origine. Cette scène est bel et bien une élaboration intériorisée du mythe, où la réflexion spéculaire évolue en une projection psychologique. L'identité révélée par le visage de l'eau est une identité mouvante, variable, chaotique, indéfinie, plurielle... Elle apparaît sous la forme de l'eau elle-même, du visage changeant ou métamorphosé, bref d'une figure sans traits à habiter, à incarner.

18 Jacques est comme le Narcisse de la poétique baroque, il se partage entre la fuite et le reflet, puisqu'au miroir de l'eau, le reflet est instable et peu rassurant. Son image réfléchie se présente constamment en fuite et insaisissable. Le support de l'eau est, en ce sens, propre à l'évanouissement et Narcisse est, de ce fait, soumis à l'inquiétude et au danger. Le reflet de Jacques est le double de ce qui est reflété. C'est une sorte de reconnaissance de soi-même, mais c'est aussi une identité reproduite, autre. Dans la légende, Narcisse, à force de se regarder, se vide de son essence par cette contemplation du double dans le reflet. Fasciné par les visions multiples que l'eau lui renvoie, Jacques reste lui aussi malgré tout paralysé par la peur de la fuite de son image et ne peut se permettre le moindre mouvement qui risque de le perdre à jamais :

Cette image de lui-même sur laquelle il se penche, Narcisse ne trouve pas dans sa ressemblance une sécurité suffisante. Ce n'est pas l'ombre stable du miroir [...] [mais] une image fuyante, une image en fuite, car l'élément qui la porte et la constitue est voué par essence à l'évanouissement. L'eau est le lieu de toutes les traîtrises et de toutes les inconsistances : dans le reflet qu'elle lui propose, Narcisse ne peut se reconnaître sans inquiétude, ni s'aimer sans danger. En lui-même, le reflet est un thème équivoque : le reflet est un double, c'est-à-dire à la fois un autre et un même. . $^{38}$

19 La fuite, la confusion et l'ambiguïté sont propres au reflet des substances liquides mais elles sont impossibles pour le reflet fixe, identique et symétrique au miroir. La rêverie par la fuite de l'image intervient dès qu'un élément perturbant vient troubler le calme de la surface de l'eau, en ridant sa surface et par conséquent en faisant onduler l'image reflétée sur l'eau. L'image de Jacques renvoyée par l'eau mouvante et instable est ainsi une image flexible, déformée, fictivement symétrique qui accueille ses fantasmes. Évanescente par excellence, l'image est dérobée par le courant de l'eau et s'enfuit avec elle. Cette vision du courant qui emporte avec lui notre image ressemble bien à celle que Virginia Woolf reproduit dans son roman d'exploration du «flux de conscience », Les Vagues 
(1931) : «Je vais me laisser porter par l'élan général. Ma pensée en surface glisse comme un fleuve gris pâle reflétant ce qui passe. Je ne me souviens pas de mon passé, ni de mon nez, ni de la couleur de mes yeux, ni de l'opinion que j'ai de moi $»^{39}$. Jacques s'exprime quasiment par le même monologue intérieur qui l'avale vers les profondeurs de l'eau, le vouant à la mort. Dans ces conditions, englouti dans les abîmes de l'élément liquide, l'autre disparaît et meurt à jamais. L'écoulement de l'eau est à percevoir justement comme ce fatal écoulement du Moi au miroir de la projection de cet autre qui nous habite.

La représentation du Narcisse du Monde désert est celle d'une image plus mentale que physique, une pseudo-sublimation ${ }^{40}$ qui mêle réalité et sur-réalité ${ }^{41}$. La mort est ainsi perçue comme le franchissement d'une porte étroite ${ }^{42}$, pour reprendre le titre de Gide, un moyen de salut et de libération qui réconcilie Jacques et l'Unité du monde. À présent, pour lui «Le narcissisme est l'effacement de la trace de l'autre dans le désir de l'Un »43. Son geste éclatant unit chair et esprit et noie à jamais le sens de culpabilité qui le tenaille :

Nu-tête et sans pardessus. Nuit de novembre [...]. Il prend le quai du Rhône [...]. Personne dans les rues [...]. Il parle à voix haute pour exprimer l'afflux de ses pensées. Je suis fort, il fait froid. Comment ai-je attendu jusqu'à ce soir ? Que c'était long de me trouver! La porte à franchir est étroite. La porte dure et abjecte est étroite. Elle n'est pas vraie. De l'autre côté sera ce que je désire. Dieu tu m'as donné ce grand désir. Dieu tu te connais dans mon désir. Ma vie fut division sans Dieu et ce soir je fais l'Unité. [...] On voit Jacques regarder le Rhône. Une lumière électrique jette l'ombre de Jacques sur le quai. Le fleuve est noir, boursouflé, un courant formidable [...] c'est le Rhône. Ce soleil ne paraitra jamais. (MD, p. 155-156)

L'eau acquiert sans aucun doute une valeur symbolique et elle nous apparait dès lors comme source de mort régénératrice qui permet une communion mystique avec Dieu. Finalement, englouti par les eaux du Rhône, Jacques n'est plus deux mais Un. D'autre part, quelques pages plus loin, il nous présente cette mort comme des « fiançailles » (MD, p. 158), ses noces mystiques avec Dieu, une sorte d'union amoureuse, un don de soi qui le ramène à nouveau à la rêverie du lac Léman ( $M D, p .13-14)$, puisque Jacques voit, juste avant de se noyer, l'image de l'eau « comme jadis quand [il] étai[t] gosse » (MD, p. 158).

Ainsi qu'il l'annonçait dans son «envie de la mort» (MD, p. 86), Jacques accomplit son suicide en se jetant dans l'eau pour rejoindre à la fois Dieu, une étreinte maternelle rassurante, "lui-même et l'image-reflet, mourant tel Narcisse réuni avec l'objet de son amour $»^{44}$. La mort du héros a lieu dans sa « ville natale, maternelle» (MD, p. 157), et nous est décrite comme une entrée dans un « sein merveilleusement doux, éternel » (MD, p. 86) qui s'ouvre et dans lequel Jacques entre irrémédiablement pour s'y retrouver. De toute évidence, comme nous l'avons dit, la mort dans l'eau se révèle comme un état de régression prénatale, un état de non-vie, qui reproduit l'étrange situation intra-utérine du ventre maternel. Par la noyade, le héros du Monde désert fait alors un retour à l'état « océanique » dont parlait Freud.

En s'appuyant sur des textes de la seconde topique freudienne, Jouve retrouve ainsi une universalité du mythe de Narcisse. Il n'y parle plus seulement de la libido du sujet se contemplant elle-même dans une relation sans autre, mais au contraire d'un éros qui pousse le sujet à s'oublier lui-même et à laisser parler l'Autre. C'est ainsi que Narcisse devient le patron mystique de l'écrivain, et se transforme à nouveau, comme en des temps plus anciens, en une métamorphose de l'artiste. Sans doute cette lecture jouvienne du mythe, mêlant mysticisme religieux et psychanalyse, n'aurait-elle pas eu lieu sans Blanche Reverchon ${ }^{45}$. Pour preuve : c'est elle encore qui introduira un autre écrivain, 
Henry Bauchau (1913-2012), à cette relecture des mythes anciens conciliant introspection et conversion religieuse.

\section{NOTES}

1. Cf. J. Bellemin-Noël, «Lire l'Homme ", dans Psychanalyse et littérature, Paris, Quadrige/PUF, «Essai », 2002, p. 83-114.

2. Cf. Ovide, Les Métamorphoses, III, 339-510, tr. fr. Georges Lafaye, Paris, Gallimard, «folio classique », p. 117-123.

3. Cf. H. Damisch, "D'un Narcisse l'autre », dans "Narcisses", Nouvelle revue de psychanalyse, n. 13, Paris, Gallimard, printemps 1976, p. 109-146.

4. Cf. S. Nicolas, Histoire de la psychologie française : naissance d'une nouvelle science, Paris, In Press, 2002.

5. «Les dieux, les mythes font partie de sa réalité [de l'homme] et forment aussi son gigantesque château de cartes ", P. J. Jouve, "Inconscient, spiritualité et catastrophe », dans Commentaires, Neuchâtel, Éditions À la Baconnière, 1950, p. 32.

6. L'histoire du Monde désert se déroule principalement en Suisse, en février 1911. Là, deux amis, Jacques et Luc, partagent un chalet et font la connaissance de Baladine, une très belle jeune femme russe divorcée. Jacques est lui aussi un très bel homme et la jeune fille en tombe éperdument amoureuse. Épris de sa beauté physique et de la nature des Alpes qui l'entourent, Jacques vit dans un total épanouissement de soi : il skie nu, attend les premier rayons de soleil... mais attrape malheureusement une pneumonie. Baladine s'occupe de lui et ne le quittera désormais plus. Bisexuel, Jacques avoue être particulièrement attiré par les jeunes hommes et provoque un scandale lors d'une rencontre amoureuse avec un jeune garçon du voisinage, une petite fripouille appelée Charles Stoebli. Jacques subit alors une sorte de chantage et, pour échapper aux rumeurs, retourne à Genève. Malgré la fréquentation des garçons, Baladine choisit de vivre quand même avec lui et pose comme modèle pour ses œuvres. Luc, amoureux lui aussi de Baladine, fait retour chez le couple. Séduite par ces deux hommes, la jeune russe gère très rationnellement et "fidèlement" son ménage à trois... Affligé par la culpabilité de son homosexualité, Jacques sombre dans la dépression et sera trouvé mort noyé. La guerre survient et quatre ans plus tard, Baladine devient une femme d'affaires qui vit seule avec le petit Pierrot, le fils de Jacques. Elle retrouve Luc, accepte de l'épouser mais disparaît bizarrement aussitôt. Luc la recherchera en vain à Paris, s'enfonçant dans le plus grand désespoir. Le Monde désert parle ainsi des rapports difficiles entre la vie amoureuse et la création artistique chez trois personnages : Jacques de Todi, homosexuel et jeune peintre genevois, Luc Pascal, le poète maudit (alter ego de P. J. Jouve avec qui la ressemblance physique est notable) et la mystérieuse Baladine, femme de Jacques, qui pousse les hommes qu'elle aime à se réaliser sans les protéger de la mort physique ou symbolique. Le roman se lit à deux niveaux: la vie visible, extérieure, des personnages et leur vie intérieure, pernicieuse et secrète, que Jouve nous révèle petit à petit et d'une manière presque angoissante au fil du texte.

7. Il est fort probable que notre auteur ait lu l'ouvrage de Gide qui était à l'époque, rappelons-le, bien plus qu'un ami, une véritable icône de la littérature et de la vie culturelle françaises.

8. « Au bord du fleuve du temps, Narcisse s'est arrêté. Fatale et illusoire rivière où les années passent et s'écoulent. Simples bords, comme un cadre brut où s'enchâsse l'eau, comme une glace 
sans tain ; où rien ne se verrait derrière ; où, derrière, le vide ennui s'éploierait. Un morne, un léthargique canal, un presque horizontal miroir ; et rien ne distinguerait de l'ambiance incolorée cette eau terne, si l'on ne sentait qu'elle coule. [...] [M]aintenant, il se penche [...] Et voici que, comme il regarde, sur l'eau soudain une mince apparence [...] toute une fuite de rapides images qui n'attendaient que lui pour être, et qui sous un regard se colorent [...] [comme des] visions qui selon le cours des eaux ondulent, et que les flots diversifient. Narcisse regarde émerveillé ; - mais ne comprend pas bien [...] si son âme guide le flot, ou si c'est le flot qui la guide ", A. Gide, Le Traité du Narcisse, dans Le retour de l'enfant prodigue, Paris, Gallimard, « Folio », 1912, p. 12-13. Ce traité parut dans Entretiens politiques et littéraires, en janvier 1891.

9. Il précise que ceux-ci «[...] se prennent eux-mêmes comme objet sexuel; ils partent du narcissisme et recherchent des jeunes gens qui leur ressemblent qu'ils puissent aimer comme leur mère les a aimés eux-mêmes ", S. Freud, Trois essais sur la théorie de la sexualité (1905), tr. fr. Blanche Reverchon-Jouve, Paris, Gallimard, «Idées », 1962, p. 167-168, note 13. En 1910, Freud rédige «Le Président Schreber : remarques psychanalytiques sur un cas de paranoïa » où il fait apparaître pour la première fois la notion de narcissisme. Cf. S. Freud, « Le Président Schreber ", dans Cinq psychanalyses, tr. fr. M. Bonaparte et R.M. Lœwenstein, Paris, PUF, 1954.

10. Ce terme a été introduit "par Freud pour distinguer deux modes d'investissement de la libido : celle-ci peut prendre comme objet soit la personne propre (libido du moi ou narcissique), soit un objet extérieur (libido d'objet)», J. Laplanche et J.-B. Pontalis, Vocabulaire de la psychanalyse, sous la dir. de Daniel Lagache, Paris, Quadrige / PUF, 1967 ; 2011 pour la présente édition, p. 226.

11. On utilise le terme "narcissique [...] pour désigner le fait qu'un individu dans son ensemble maintient sa libido dans le moi sans rien en dépenser dans des investissements d'objets ", S. Freud, Au-delà du principe du plaisir (1920), dans Essais de psychanalyse, tr. fr. Jean Laplanche et J.-B. Pontalis, Paris, Éditions Payot \& Rivages, 2001, p. 108.

12. J. Laplanche et J.-B. Pontalis, Vocabulaire de la psychanalyse, cit., p. 262.

13. S. Freud, Pour introduire le narcissisme (1914), tr. fr. Olivier Mannoni, Paris, Éditions Payot \& Rivages, 2012, p. 38.

14. J. Laplanche et J.-B. Pontalis, Vocabulaire de la psychanalyse, cit., p. 272.

15. Ce terme apparaît pour la première fois dans une lettre du 5 décembre 1927 de Romain Rolland pour définir une sensation d'éternité, une dimension sans frontières, sans bornes et donc "océanique ", qui exprime fondamentalement une volonté de faire un avec le monde. Freud, son ami, en propose une interprétation comme un moi primaire, typique du nourrisson qui ne distingue pas entre son moi et le monde extérieur. Ce moi primaire lié à la nostalgie de la vie enfantine se juxtapose au sentiment du moi de la maturité. Cf. S. Freud, Le Malaise dans la culture (1929), dans Euvres complètes, 1912-1930, t. XVIII, Paris, PUF, 1994, p. 258.

16. P. J. Jouve, «Inconscient, spiritualité et catastrophe », cit., p. 28.

17. Sylvie Poza nous fait « remarquer [...] à propos du nom de Jacques [que] Jacopo ou Jacopone da Todi était un poète mystique italien du treizième siècle qui, après la mort de celle qu'il aimait, devint moine franciscain et vécu en ascète. Jacques a, lui [aussi], perdu sa bien-aimée très jeune et $\mathrm{a}$, dès lors, essayé d'une vie d'ascète et d'artiste ", S. Poza, Lecture critique des romans de Pierre Jean Jouve, Fleury-sur-Orne, Librairie Minard, 1994, p. 73. Nous savons aussi que « Jacques porte le prénom d'un apôtre qui mourut martyr, et [que] son nom de famille [de Todi] évoque, par un jeu homonymique, le taudis, taudis moral plus que matériel dont il est issu, désert des sentiments et de tendresse que lui offre sa famille», B. Bonhomme, «Le jeux de l'écriture dans l'œuvre de Pierre Jean Jouve ", dans Ch. Blot-Labarrère (dir.), Pierre Jean Jouve 5 - Jouve et les jeux de l'écriture, Paris, Lettres Modernes Minard, 1996, p. 136. Italiques de l'auteur.

18. Sur les deux pulsions, voir S. Freud, Le Moi et le Ça (1923), dans Essais de psychanalyse, tr. fr. Jean Laplanche, Paris, Éditions Payot \& Rivages, 2001, p. 282. 
19. «Le moi est avant tout un moi corporel, il n'est pas seulement un être en surface, mais il est lui-même la projection d'une surface [...]. Il peut ainsi être considéré comme une projection mentale de la surface du corps, et de plus, [...] il représente la surface de l'appareil mental », Ibid., p. 264 et note 5.

20. K. Shärer, Thématique et poétique du mal dans l'œuvre de Pierre Jean Jouve, Paris, Lettres Modernes Minard, 1984, p. 7-9. Italiques de l'auteur.

21. R. Micha, La scène capitale de Jouve, Bruxelles, La maison du poète, 1942, p. 12-15.

22. " Eh bien, sois ce que tu es par art aussi bien que par nature, selon le vers de Shakespeare. Lietoi avec un homme ayant comme toi l'amour "du même". Abandonne l'opposition qui t'épuise. Sépare-toi de la morale et de ta famille ", P. J. Jouve, Le Monde désert, Paris, Mercure de France, 1960 , p. 81 (dorénavant MD). Italiques de l'auteur.

23. "Mon père était un homme de Dieu ", Ibid., p. 17, ou encore à propos des habitants de Bâle : "Ah! leur théologie vous pince comme un casse-noisette entre la grâce et les vertus, rien à faire, vous êtes pris, c'est la vie dans la Bible. Toutes mes difficultés viennent de là. Tous les empêchements de vivre, les fausses démarches, les malaises ", Ibid., p.18. C'est pourquoi "Jacques ne croit plus en "un Dieu" (ni celui de son père, ni celui de sa tante Pauline, ni le Jehovah du Temple ni le Seigneur que l'on prie en secret). Nous les enfants des cavernes nous serons les adorateurs du Soleil. À Jésus-Christ, préférons le totem du Feu », Ibid., p. 25-26.

24. «[...] maîtres et autorités ont continué le rôle du père ; leurs ordres et leurs interdictions sont restés puissants dans le moi-idéal et, sous forme de conscience morale, exercent désormais la censure morale. La tension entre les exigences de la conscience morale et les réalisations du moi est ressentie comme sentiment de culpabilité ». S. Freud, Le Moi et le Ça, cit., p. 278. Italiques de l'auteur.

25. W. Rupolo, Pierre Jean Jouve le roman comme "refoulement théâtral », Fleury-sur-Orne, Librairie Minard, 1997, p. 38.

26. Ibid., p. 16.

27. "La rigueur puritaine de Genève et de la Suisse, vues par Jouve, éclairent cruellement Le Monde désert qui est le plus dépouillé et le plus aride des romans », S. Sanzenbach, Les romans de Pierre Jean Jouve. Le romancier en son miroir, Paris, Librairie Philosophique J. Vrin, 1972, p. 42-43.

28. «Le sentiment de culpabilité normal, conscient (conscience morale) [...] repose sur la tension entre le moi et l'idéal du moi et il est l'expression d'une condamnation du moi par son instance critique. [...] Dans la mélancolie l'impression que le sur-moi s'est annexé la conscience est encore plus forte. Mais ici le moi n'élève aucune prestation, il se reconnait coupable et se soumet aux châtiments. [...] dans la mélancolie, l'objet qui s'attire la colère du sur-moi est englobé par identification dans le moi », S. Freud, Le Moi et le Ça, cit., p. 295-296.

29. J. Decottignies, Pierre Jean Jouve romancier ou l'expérience de l'abîme, Paris, Librairie José Corti, 1994, p. 79.

30. «Besoin d'évasion, avant tout du réel : la rêverie représente un refuge, le moyen d'échapper aux interdits familiaux, un substitut de la révolte », W. Rupolo, op. cit., p. 78.

31. Cf. S. Freud, Au-delà du principe du plaisir, cit., p. 82.

32. Pierre Jean Jouve s'exila en Suisse pendant la seconde guerre mondiale et devint un acteur important de la résistance intellectuelle contre le nazisme avec ses poèmes apocalyptiques de Gloire et de La Vierge de Paris.

33. À l'époque, La Porte étroite d'André Gide (1911) fait tendance pour son analyse psychologique et est certainement un modèle que poursuit Jouve. L'œuvre sera citée en filigrane vers la fin du roman quand la mort de Jacques à travers le suicide sera racontée comme le passage à travers " une porte étroite».

34. Luc Pascal, qui ressemble fortement à Jouve, nous est présenté dans le roman comme un écrivain célèbre, parisien, qui était « déjà chauve [et] plutôt sec » (MD, p. 32).

35. «L'amour modèle l'instinct à son image » (MD, p. 44). 
36. Jouve-écrivain et son monde réel ne sont pas à confondre avec le narrateur et le monde imaginé du roman. En ce sens, le plan fictionnel de l'œuvre est à distinguer fortement du monde social réel auquel notre auteur appartient.

37. « le petit personnage assis et cherchant à comprendre le sens d'une histoire aussi sombre, bien qu'il soit féminin c'est lui-même » ( $M D$, p. 15).

38. G. Genette, «Complexe de Narcisse », dans Figures I, Paris, Éditions du Seuil, 1966, p. 28. Italiques de l'auteur.

39. V. Woolf, Les Vagues, tr. fr. Michel Cusin, Paris, Gallimard, 2012, p. 157.

40. «[...] toute sublimation ne se produit-elle pas par l'intermédiaire du moi qui commence par transformer la libido d'objet sexuel en libido narcissique, pour lui assigner éventuellement ensuite un autre but? ", S. Freud, Le Moi et le Ça, cit., p. 270.

41. La perte du contact avec la réalité s'effectue : «[...] quand il avait complètement perdu de vue toute autre réalité » $(M D$, p. 16). Un dialogue muet s'établit alors entre Jacques et le visage, une sorte de fiction symétrique, au-delà de la parole, proprement apophatique, car «sans employer de mots, [il veut] lui parler ", Ibidem.

42. «La porte, ou le seuil, est à la fois un symbole de séparation, de frontière, et de communication, de passage. [...] Si bien que l'acte, en étant métaphorisé et métonymisé par l'ouverture de la porte, en vient à figurer une autre ouverture de porte qui symbolise, celle-là, le passage initiatique à un autre état du sujet (à la fin de ses épreuves, le néophyte jouit d'une toute autre existence qu'avant l'initiation: il est devenu un autre). Ainsi l'union avec le double constitue une expérience initiatique, elle correspond à une transformation du sujet, lequel se voit unifié, purifié », S. Poza, op. cit., p. 97.

43. Cf. A. Green, «Le narcissisme primaire : structure ou état» (1967), dans Narcissisme de vie, narcissisme de mort, Paris, Les Éditions de Minuit, 2007. André Green (1927-2012), élève de Lacan, depuis 1963, est un des rares auteurs - sinon le seul - à avoir tenté d'articuler la théorie du narcissisme avec celle de la dernière théorie des pulsions. Alors que le narcissisme n'est généralement envisagé que sous ses aspects positifs, par lesquels on le rattache aux pulsions sexuelles de vie, il montre la nécessité de postuler l'existence d'un narcissisme de mort, qu'il appelle le narcissisme négatif. À la différence du premier, qui vise l'accomplissement de l'unité du Moi, le second tend au contraire à son abolition dans l'aspiration au zéro.

44. S. Poza, op. cit., p. 136.

45. Cf. H. Bauchau, Pierre et Blanche. Souvenirs sur Pierre Jean Jouve et Blanche Reverchon, textes présentés et assemblés par Anouck Cape, Arles, Actes Sud, 2012. Henry Bauchau estime que Blanche Reverchon a sauvé la vie de son époux qui avait des tendances suicidaires. Nous apprenons qu'à partir de 1921, Blanche « rééduque » Pierre Jean Jouve : elle prend soin de ses angoisses, soigne sa neurasthénie et lui fait découvrir la psychanalyse, la mystique, en l'amenant à retrouver sa vraie voie : l'écriture. Elle aime les romans, et Jouve, sur ses conseils, en écrit quelques-uns : Paulina 1880, Le Monde désert, Hécate et La Scène capitale.

\section{INDEX}

Mots-clés : Jouve (Pierre Jean), narcissisme, Monde désert, Freud (Sigmund), psychanalyse, Moi (déchirement du), Autre (quête de l'), image reflétée, identité plurielle, unité (désir de l'), communion avec Dieu 